\title{
Assessment of bronchiectasis by computed tomography
}

\author{
IM MOOTOOSAMY, RH REZNEK, J OSMAN, RSO REES, MALCOLM GREEN
}

From the Departments of Diagnostic Radiology and Chest Medicine, St Bartholomew's Hospital, London

ABSTRACT Computed tomography and bronchography were used to assess the distribution of bronchiectasis in 15 lungs from eight patients with clinical features of the disease. Of the 36 lobes adequately displayed by bronchography, 22 were found to have bronchiectasis and 14 were found to be normal by both techniques. Cystic disease was readily identified by computed tomography but the cylindrical and varicose types of bronchiectasis could not be distinguished. Segmental localisation was less accurate, with agreement between computed tomography and bronchography in 116 out of 130 segments. It is concluded that with a modern high resolution scanner computed tomography provides a useful method of assessing lobar distribution in bronchiectasis.

The incidence of bronchiectasis in the United Kingdom has decreased since the advent of antibiotic treatment and the decline in severe respiratory disease in childhood. Nevertheless, it still occurs as the result of severe pulmonary infections and in association with immune deficiency, cystic fibrosis, and other conditions.

The disease is defined as irreversible abnormal dilatation of the bronchi and has been classified by Reid ${ }^{1}$ into three types - cystic, varicose, and cylindrical. Although the plain chest film may provide a clue, particularly in the cystic type, bronchography is still the definitive investigation. It is indicated when it is necessary to confirm the clinical diagnosis or, if the disease is localised, to exclude the disease in other parts of the lungs if lobectomy is contemplated. Bronchography is an unpleasant, potentially dangerous, ${ }^{23}$ and invasive investigation that requires considerable skill and even when expertly performed it may leave areas of lung inadequately examined owing to technical or other difficulties.

Computed tomography scanners with their improved resolution provide a potential means of detecting or excluding bronchiectasis and so avoiding bronchography. Naidich $\mathrm{et} \mathrm{al}^{4}$ described the appearances of bronchiectasis on computed tomography in six patients and recently Müller $e t a^{5}$ presented results obtained in 11 patients. Whereas Naidich et al were optimistic about computed tomography re-

Address for reprint requests: Dr IM Mootoosamy, Department of Diagnostic Radiology, St Bartholomew's Hospital, London ECIA 7BE.

Accepted 8 July 1985 placing bronchography in a substantial number, Müller et al found correspondence in only about half the lungs studied.

The purpose of this study is to assess the place of modern high resolution computed tomography scanners in the clinical management of patients with bronchiectasis and to investigate the accuracy with which lobar and segmental disease and the type of disease present can be predicted.

\section{Patients and methods}

Eight patients with a clinical diagnosis of bronchiectasis were included in the study, all of whom had posteroanterior, lateral, and supine chest films; bronchograms; and computed tomography scans. Bilateral bronchograms were obtained in seven of the patients (aqueous dionosil being used), three via a fibreoptic bronchoscope and four by transnasal tracheal intubation. In one patient a right bronchogram only was available as the examination had to be abandoned before the left side was examined owing to the patient's distress.

Computed tomography scans were performed with a GE 9800 scanner with a two second scan time, 512 matrix, and $10 \mathrm{~mm}$ scan thickness. The patients were examined supine with slices at $1 \mathrm{~cm}$ intervals through the lungs. Scans were also obtained with the patient prone where indicated to differentiate dependent pulmonary vessels. The interval between computed tomography and bronchography was less than six months in all patients except patient 1 (with cystic bronchiectasis), who was scanned at 12 months.

The chest radiographs were examined indepen- 

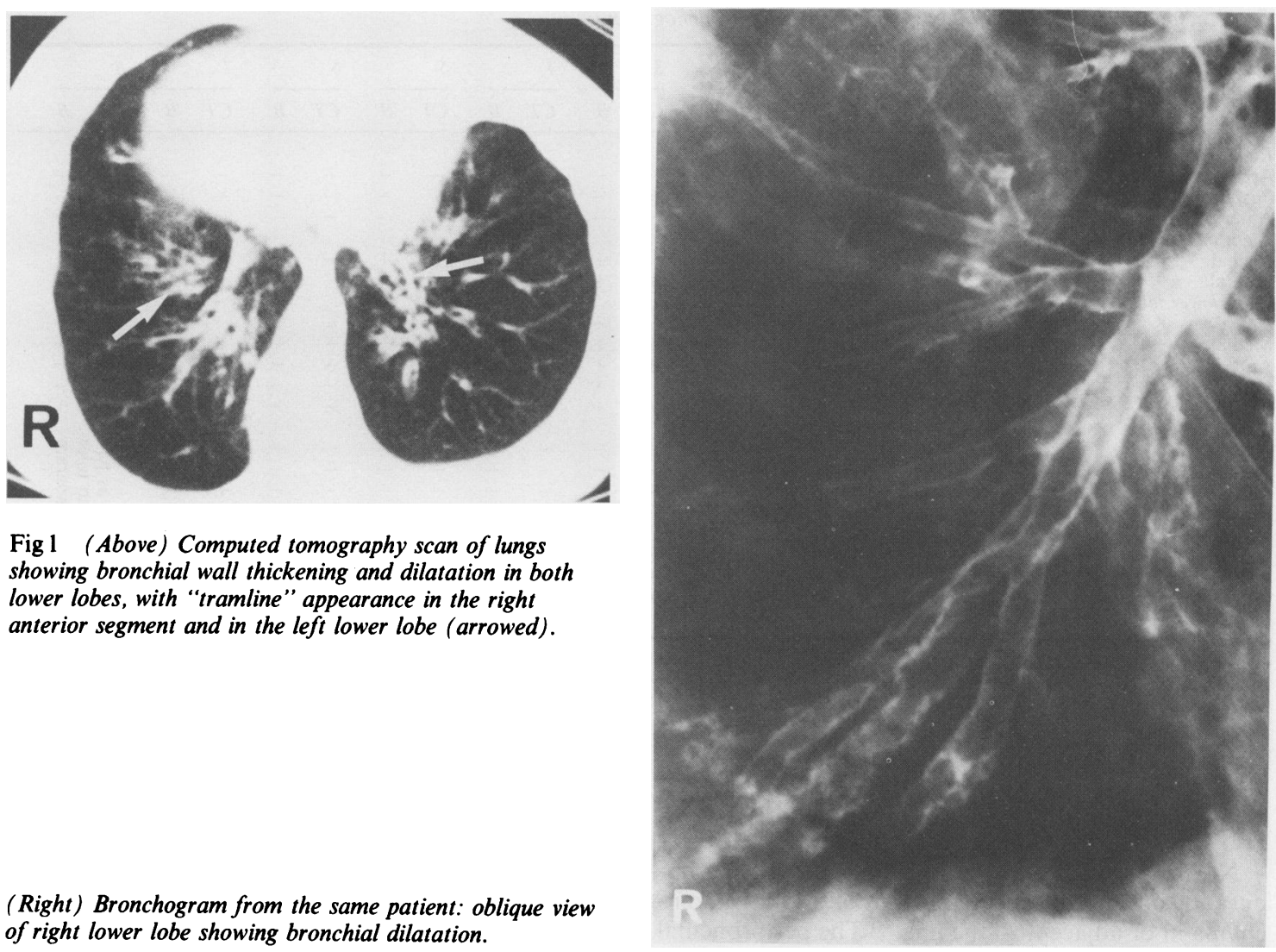

(Right) Bronchogram from the same patient: oblique view of right lower lobe showing bronchial dilatation.

dently by one radiologist (RSOR) without knowledge of other findings. The lobar distribution of changes consistent with bronchiectasis was recorded from the plain films. The presence of bronchiectasis as seen on

bronchography was recorded to include its type (cystic, varicose, or cylindrical), lobar distribution within each lung, and, within each lobe, disease of individual segments. The computed tomography scans were as-

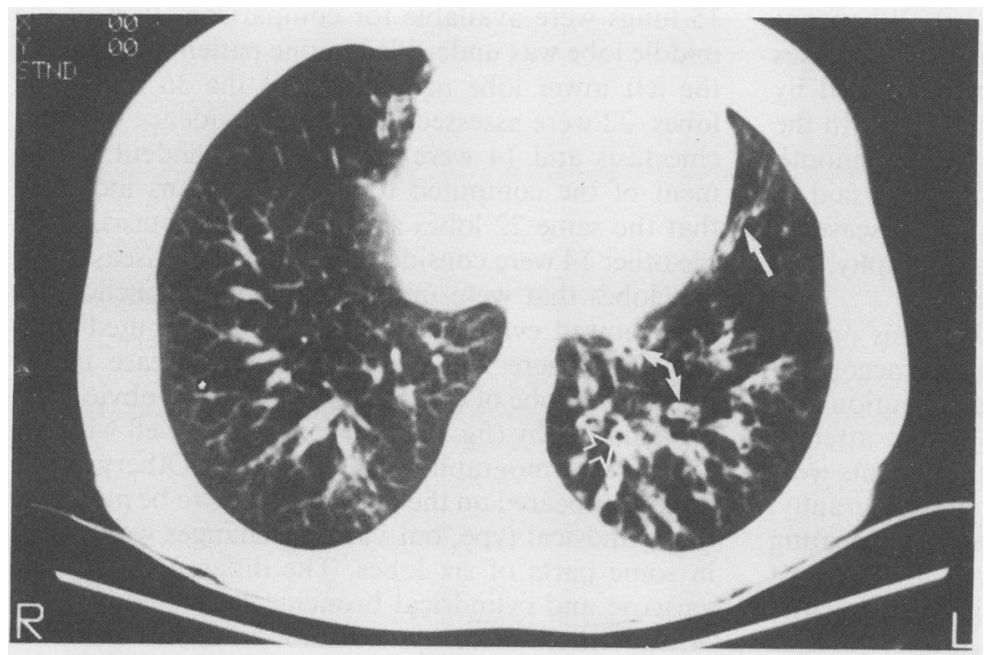

Fig 2 Computed tomography scan of lungs showing bronchial dilatation and bronchial wall thickening in the left lower lobe and in the lingula (arrowed). A dilated, thick walled bronchus cut transversely is seen in the posterior segment of the left lower lobe as a circular lucency (open arrow). 
Comparison of results of computed tomography and bronchography

\begin{tabular}{|c|c|c|c|c|c|c|c|c|c|c|c|c|c|c|c|c|}
\hline \multirow[t]{2}{*}{ Patient } & \multicolumn{2}{|l|}{1} & \multicolumn{2}{|l|}{2} & \multicolumn{2}{|l|}{3} & \multicolumn{2}{|l|}{4} & \multicolumn{2}{|l|}{5} & \multicolumn{2}{|l|}{6} & \multicolumn{2}{|l|}{7} & \multicolumn{2}{|l|}{8} \\
\hline & $C T$ & B & $C T$ & $\boldsymbol{B}$ & $C T$ & $\boldsymbol{B}$ & $C T$ & B & $C T$ & $\boldsymbol{B}$ & $C T$ & $B$ & $C T$ & $B$ & $C T$ & $\boldsymbol{B}$ \\
\hline \multicolumn{17}{|l|}{ Right upper lobe } \\
\hline Apical & - & $\mathbf{v}$ & - & - & - & - & - & - & - & - & - & - & - & - & - & - \\
\hline Posterior & + & - & - & - & - & - & - & - & - & - & - & - & - & - & - & - \\
\hline $\begin{array}{l}\text { Anterior } \\
\text { Right middle lobe }\end{array}$ & + & $\mathbf{v}$ & - & - & - & - & - & - & - & - & - & - & \multicolumn{2}{|c|}{$\otimes$} & - & - \\
\hline Medial & + & + & - & - & c & + & - & - & + & + & - & - & + & $\mathbf{v}$ & + & $\star$ \\
\hline Lateral & + & + & - & - & + & + & - & - & - & - & - & - & + & $\mathbf{v}$ & - & $*$ \\
\hline Right lower lobe & \multicolumn{2}{|c|}{$\otimes$} & & & & & & & & & & & \multicolumn{2}{|c|}{${ }_{\otimes}^{+}$} & & \\
\hline Apical & c & c & - & - & - & - & - & - & - & - & - & - & - & - & - & - \\
\hline Medial & c & c & + & + & + & + & - & - & - & - & - & - & + & $\mathbf{v}$ & - & + \\
\hline Anterior & c & c & - & - & 0 & + & - & $\mathbf{v}$ & - & - & - & - & + & $\mathbf{v}$ & - & - \\
\hline Lateral & c & c & - & + & - & + & + & - & - & - & - & - & + & $\mathbf{v}$ & - & - \\
\hline Posterior & c & c & + & $\mathbf{v}$ & + & + & + & $\mathbf{v}$ & + & + & - & - & + & v & + & + \\
\hline Left upper lobe & & & & & & & & & & & & & & & & \\
\hline Apical & + & - & - & - & - & - & - & - & - & - & - & - & - & - & \multirow{9}{*}{\multicolumn{2}{|c|}{ 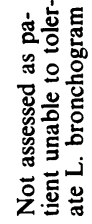 }} \\
\hline Anterior & - & - & - & - & - & - & - & - & - & - & - & - & - & - & & \\
\hline Superior lingula & - & + & + & $\mathbf{v}$ & - & - & - & - & - & - & - & - & + & - & & \\
\hline Inferior lingula & - & - & 0 & $\mathbf{v}$ & - & - & - & - & + & + & - & - & + & + & & \\
\hline Left lower lobe & & & & & & & & & & & 8 & & & & & \\
\hline Apical & - & $\mathbf{v}$ & - & - & - & $*$ & - & - & - & - & - & - & - & - & & \\
\hline Posterior & + & $\mathbf{v}$ & + & + & + & $*$ & + & + & + & + & + & + & + & + & & \\
\hline Lateral & + & $\mathbf{v}$ & + & + & + & $*$ & + & - & + & + & + & + & + & + & & \\
\hline Anterior & - & $\mathbf{v}$ & - & - & - & $*$ & + & + & - & - & + & + & + & + & & \\
\hline
\end{tabular}

$\mathrm{CT}=$ computed tomography scan; $\mathrm{B}=$ bronchogram $++=$ cylindrical bronchiectasis; $\mathrm{v}=$ varicose bronchiectasis; $\mathrm{c}=$ cystic bronchiectasis; $-=$ normal; $\Theta=$ consolidation; ${ }^{*}=$ underfilling; $\otimes=$ lobes where bronchiectasis changes seen on plain radiograph.

sessed independently by another radiologist (RHR), also without knowledge of other findings.

Normal bronchi can be seen on computed tomography only in the central portions of the lung parenchyma in or adjacent to the hila. ${ }^{46}$ The peribronchial fibrosis, however, associated with bronchiectasis ${ }^{6}$ with resultant bronchial wall thickening and dilatation renders bronchi visible in more peripheral parts of the lung, ${ }^{6}$ and thickening of the wall combined with the lack of peripheral tapering suggests the diagnosis. When cut longitudinally these abnormal bronchi appear as "tramlines" (fig 1a). When cut transversely they appear as circular or oval lucencies (fig 2) and the lack of tapering can be confirmed by examination of two or more contiguous slices. In the cylindrical type of disease the walls have a smooth outline, whereas in the varicose type they are said to have a "beaded" appearance. ${ }^{6}$ Cystic disease is readily recognisable on computed tomography, the cysts being arranged in clusters or strings.

The presence or absence of bronchiectasis on the computed tomography scans was recorded according to these criteria, and after the lobar distribution and the type of disease had been established an attempt was made to predict whether particular segments were affected within each lobe. On computed tomography, pulmonary lobes can be recognised easily by locating the main fissures. ${ }^{78}$ Segmental anatomy, although not so easy, can be identified by locating the segmental bronchi and pulmonary vessels. ${ }^{89}$

\section{Results}

The chest radiographs were thought to be normal in five patients and to show changes consistent with bronchiectasis in three. Patient 1 showed cystic changes in the right lower lobe, patient 6 showed tramline shadows in the left lower lobe, and patient 7 showed tramline shadows in the right middle and lower lobes (table).

Bronchography was performed in eight patients; in one patient only one side could be examined, so that 15 lungs were available for comparison. Part of the middle lobe was underfilled in one patient and part of the left lower lobe in another; of the 36 remaining lobes, 22 were assessed as showing evidence of bronchiectasis and 14 were normal. Independent assessment of the computed tomography scans indicated that the same 22 lobes showed bronchiectasis, while the other 14 were considered to be free of disease. The two lobes that were underfilled in the bronchogram also showed evidence of disease on computed tomography. There was extensive cystic disease in the right lower lobe of one patient, which was obvious on bronchography (fig $3 a$ ). This correlated well with the computed tomography findings (fig $3 b$ ). Otherwise the disease appeared on the bronchograms to be mostly of the cylindrical type, but varicose changes were noted in some parts of six lobes. The differences between varicose and cylindrical bronchiectasis could not be distinguished on computed tomography. Thus there 


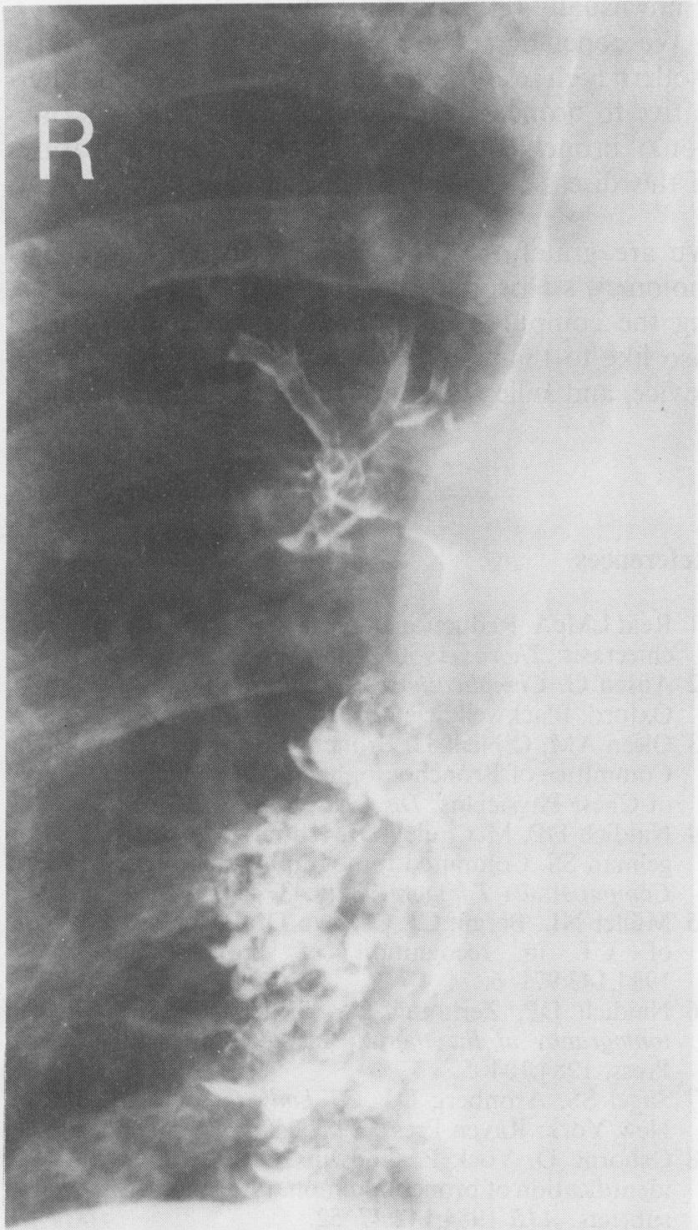

Fig 3 (a) Right lung bronchogram showing cystic bronchiectasis in the right lower lobe corresponding with the computed tomography appearances.

was concordance between the results of bronchography and computed tomography in the assessment of lobar disease by two independent observers without knowledge of each other's findings.

Agreement about segmental localisation was less good. There was agreement on the presence or absence of disease in 116 of the 130 segments: 74 were normal, and 42 abnormal. In 14 there was disagreement. Bronchiectasis could not be diagnosed on computed tomography scans in 10 of these segments (although present elsewhere in the lobe), but was thought to be present on the bronchograms; in two of these there was consolidation in which bronchiectasis could not be diagnosed. In four segments disease was attributed to a segment on the computed tomography scan but not seen in that segment on the bronchogram.

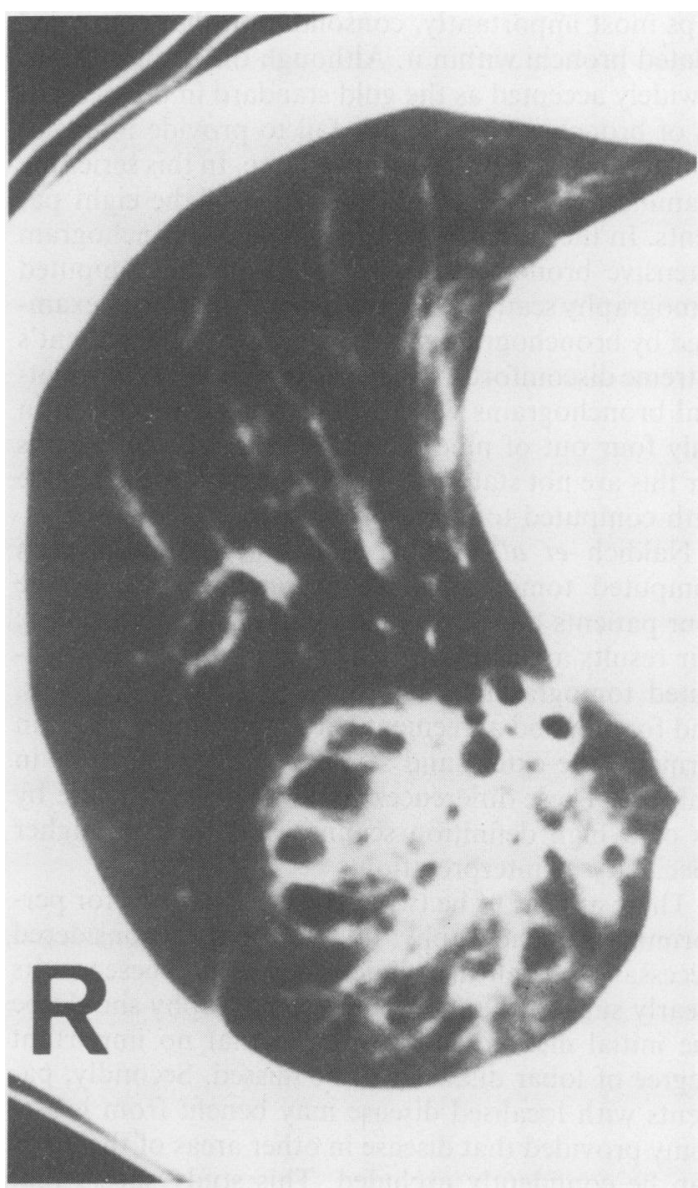

(b) Computed tomography scan of right lower lobe showing cystic bronchiectasis; there is also loss of volume.

\section{Discussion}

There appears to be no dispute that cystic bronchiectasis which is usually visible on the plain chest film, can be detected as readily by computed tomography as by bronchography. ${ }^{45}$ The results of this study suggest that cylindrical or varicose disease can also be detected or excluded on the modern scanner, as the correlation between computed tomography scan and bronchogram in assessing whether lobes were affected by bronchiectasis was excellent.

Localisation of bronchiectatic segments gave four false positive and 10 false negative results from computed tomography of a total of 130 segments. Subsequent review of these differences showed that they were due to problems in defining segmental anatomy in both techniques, distortion from fibrosis, and, per- 
haps most importantly, consolidation that concealed dilated bronchi within it. Although the bronchogram is widely accepted as the gold standard in the diagnosis of bronchiectasis, it may fail to provide information about some of the bronchial tree. In this series the examination was incomplete in two of the eight patients. In the patient with the unilateral bronchogram extensive bronchiectasis was seen on the computed tomography scan in a lower lobe of the lung not examined by bronchography (fig 2) because of the patient's extreme discomfort. In the series of Müller $e^{2}$ al $^{5}$ bilateral bronchograms were available for comparison in only four out of nine patients, although the reasons for this are not stated. Such technical failures are rare with computed tomography.

Naidich et $a l^{4}$ found good correlation between computed tomography and bronchography in the four patients they examined by these two techniques; our results are similar. Müller et $a l^{5}$ compared computed tomography and bronchography in 13 lungs, and found good agreement between the assessment in terms of the extent and severity of bronchiectasis in only six. These differences could be due to the use by us of a high definition scanner and perhaps higher specificity of interpretation.

There appear to be two clinical indications for performing bronchography: firstly, it may be considered necessary to confirm a clinical diagnosis. These results clearly suggest that computed tomography should be the initial method of choice and that no important degree of lobar disease will be missed. Secondly, patients with localised disease may benefit from lobectomy provided that disease in other areas of the lungs can be confidently excluded. This study shows that bronchiectasis of any one lobe was not missed by computed tomography and that lobar disease seen on a scan was confirmed by bronchography. Segmental localisation was less accurate but this degree of detail is not usually relevant clinically.

We conclude that computed tomography with a modern high resolution scanner is an acceptable alternative to bronchography in assessing lobar distribution of bronchiectasis in patients with clinical features of this disease.

We are grateful to the special trustees of St Bartholomew's Hospital for their generosity in purchasing the computed tomography scanner. We would also like to thank Dr I Kelsey Fry for his help and advice, and Julie Jessop for her secretarial assistance.

\section{References}

1 Reid LMcA. Reduction in bronchial subdivision in bronchiectasis. Thorax 1950;5:233-47.

2 Ansell G. Complications in diagnostic radiology. 1st ed. Oxford: Blackwell Scientific Publications, 1976:287, 289.

3 Olsen AM, O'Neill JJ. Bronchography-a report of the Committee of Bronchoesophagology, American College of Chest Physicians. Dis Chest 1967;51:663-8.

4 Naidich DP, McCauley DI, Khouri NF, Stitik FP, Siegelman SS. Computed tomography of bronchiectasis. $J$ Comput Assist Tomogr 1982;6:437-44.

5 Müller NL, Bergin CJ, Ostravo DN, Nichols DM. Role of CT in recognition of bronchiectasis. $A J R$ 1984;143:971-6.

6 Naidich DP, Zerhouni EA, Siegelman SS. Computed tomography of the thorax. 1st ed. New York: Raven Press, 1984:104-5.

7 Sagel SS, Aronberg DJ. CT body tomography. 1st ed. New York: Raven Press, 1982:64-5.

8 Osborne D, Vock P, Godwin JD, Silverman PM. CT identification of bronchopulmonary segments: 50 normal subjects. $A J R$ 1984;142:47-52.

9 Naidich DP, Terry PB, Stitik FP, Siegelman SS. CT of the bronchi. 1. Normal anatomy. J Comput Assist Tomogr 1980;4:746-53. 